\title{
GERAÇÃO DE CENÁRIOS DE VELOCIDADE DO VENTO COM MODELAGEM AUTORREGRESSIVA PERIÓDICA ASSOCIADA À MSSA
}

\author{
Moisés Lima de Menezes \\ Departamento de Estatística - Universidade Federal Fluminense \\ Rua Mário Santos Braga, S/N, Campus Valonguinho, Centro, Niterói - RJ, 24.220-900. \\ moises_lima@msn.com \\ Keila Mara Cassiano \\ Departamento de Estatística - Universidade Federal Fluminense \\ Rua Mário Santos Braga, S/N, Campus Valonguinho, Centro, Niterói - RJ, 24.220-900. \\ keilamath@hotmail.com \\ Reinaldo Castro Souza \\ Departamento de Engenharia Elétrica - Pontifícia Universidade Católica do Rio de Janeiro \\ Rua Marquês de São Vicente, 225, Gávea, Rio de Janeiro - RJ, 22.451-900. \\ reinaldo@ele.puc-rio.br \\ José Francisco Moreira Pessanha \\ Instituto de Matemática e Estatística - Universidade do Estado do Rio de Janeiro \\ Rua São Francisco Xavier, 524, Maracanã, Rio de Janeiro - RJ, 20.550-013. \\ professorjfmp@hotmail.com
}

\begin{abstract}
Resumo
Singular Spectrum Analysis (SSA) é uma técnica que decompõe uma série temporal em componentes tais como tendência, harmônicos e ruídos. Deixando de fora as componentes ruidosas e somando as outras, a série temporal pode ser suavizada. A geração de Cenários através do modelo autorregressivo periódico PAR(p) vem sendo amplamente utilizada em modelagem de séries de Energia Natural Afluente. Este artigo apresenta uma abordagem deste método de decomposição, na qual a modelagem PAR (p) é aplicada na série suavizada obtida pela SSA e por sua versão multivariada MSSA. Na ocasião são gerados 200 cenários de velocidade do vento para um horizonte de previsão de 60 meses. Para ilustrar a metodologia proposta, foram consideradas séries de velocidade do vento registradas em duas localidades na região Nordeste do Brasil. Os resultados obtidos mostram que tanto a modelagem PAR(p) quanto a geração de cenários são beneficiados pela suavização prévia da série temporal por SSA/MSSA.
\end{abstract}

Palavras Chave: MSSA, modelagem PAR(p), velocidade do vento, geração de cenários.

\begin{abstract}
Singular spectrum analysis (SSA) is a technique that decomposes a time series into a set of components, such as, trend, harmonics, and residuals. Leaving out the residual components and adding up the others, the time series can be smoothed. The scenarios generations through $\operatorname{PAR}(p)$, periodic autoregressive models, has been broadly used in modeling Affluent natural Energy series. This article presents an approach of this decomposition method, in which the PAR (p) model is applied to the smoothed series obtained by SSA and its multivariate version, named MSSA. In this case, 200 scenarios are generated for a forecasting horizon of 60 months. To illustrate the application of the proposed methodology were considered wind speed time series recorded at two locations in northeastern Brazil. The results show that both the PAR (p) model and scenarios generations are favored by prior smoothing of the time series by SSA / MSSA.
\end{abstract}

Keywords: MSSA, PAR(p) modeling, wind speed, scenarios generation. 


\section{INTRODUÇÃO}

Com o advento da energia eólica, a operação dos sistemas elétricos deverá contar com modelos capazes de prever a produção de energia elétrica nos parques eólicos, a fim de proporcionar uma integração segura e econômica dos parques na operação do Sistema Interligado Nacional (SIN). Este trabalho descreve uma aplicação do procedimento Singular Spectrum Analysis (SSA), combinado com o modelo periódico autorregressivo PAR (p), na modelagem e geração de cenários da média mensal da velocidade do vento.

SSA é um poderoso método para análise e previsão de séries temporais e incorpora elementos de análise de séries temporais clássica, estatística multivariada, geometria multivariada, sistemas dinâmicos e processamento de sinais (GOLYANDINA et al., 2001). SSA podem ser aplicados em vários campos da matemática e da física à economia e matemática financeira, meteorologia e oceanografia para ciências sociais (HASSANI, 2007).

Com base na decomposição em valores singulares (singular value decomposition - SVD), o método SSA decompõe uma série de tempo em uma soma de componentes, tal que em cada componente se concentra uma parte da energia contida na série temporal. Além disso, um pequeno conjunto de componentes ocorre na maior parte da estrutura de autocorrelação contida na série temporal, enquanto que os restantes podem ser considerados como componentes de ruído. Portanto, o conjunto de componentes pode ser classificado em dois grupos mutuamente exclusivos e exaustivos: sinal e ruído. O primeiro grupo contém os componentes que possuem algumas informações sobre a estrutura da série, enquanto o último abrange os componentes de ruído. Assim, uma versão suavizada da série temporal pode ser obtida pela soma das componentes de sinal. Tradicionalmente, uma série de tempo pode ser expressa como a soma de componentes: tendência, harmônica e ruído. A remoção do componente de ruído pode contribuir para uma melhor identificação do processo estocástico subjacente da série temporal e, conseqüentemente, melhorar a precisão das previsões. JAYAWARDENA \& GURUNG (2000) mostram que a remoção de ruídos em série hidrológica melhora a precisão das previsões. Uma boa revisão do assunto é apresentada em (ELSHORBAGY et. al, 2002).

Multi-channel Singular Spectrum Analysis (MSSA) é a extensão natural do SSA para uma série temporal multivariada. Os procedimentos de SSA e MSSA oferecem um método de prétratamento de dados. Portanto, ambos os processos podem ser utilizados a fim de melhorar o ajuste de modelos de previsão e ajudar a melhorar a precisão das previsões (HASSANI \& ZHIGLJAVSKY, 2009). Mas quando o interesse reside na previsão de um conjunto de séries temporais múltiplas qual procedimento é o mais adequado? Temos de aplicar o procedimento de SSA para cada série temporal individualmente ou aplicamos o procedimento MSSA ao conjunto de séries temporais? Para responder as perguntas acima, neste artigo são apresentados os resultados de um experimento computacional onde os procedimentos de SSA e MSSA foram aplicados a séries temporais de média mensal de velocidade do vento em Petrolina e Pesqueira, ambos localizados na região do Nordeste.

Em seguida, o modelo auto-regressivo periódico PAR (p), foi adaptado para cada série temporal suavizada obtida por SSA e MSSA. Os resultados mostram que a SSA contribui para o ajuste de PAR(p) no modelo de cada série velocidade do vento, porém a contribuição da MSSA é ainda maior. A opção pelo modelo PAR(p) deve-se ao seu uso nos modelos computacionais que apóiam o Planejamento da Operação de Médio e Curto prazo do SIN (MACEIRA et al,, 2006).

Associada aos modelos PAR(p) está a geração de cenários. Para que esta geração de cenários seja possível, a série de ruídos removida na filtragem SSA e MSSA é realocada através de um processo Bootstrap. Os cenários gerados são compostos de duas partes: uma gerada a partir da distribuição lognormal dos resíduos do modelo PAR(p) - MSSA/SSA e outra da inclusão dos ruídos removidos no processo SSA/MSSA através do processo Bootstrap. 
O presente artigo está organizado em seis seções. A seguir, na seção 2, há uma breve descrição da SSA. Na seção 3 tem-se uma descrição resumida da MSSA. A modelagem PAR(p) é introduzida na seção 4. A geração de cenários é apresentada na seção 5. Na seção 6 está a proposta deste artigo e na seção 7 está o estudo de caso com os resultados obtidos. Enfim, na seção 8, são apresentadas as principais conclusões do trabalho.

\section{SINGULAR SPECTRUM ANALYSIS}

A versão básica do método SSA pode ser dividida em duas etapas: decomposição e reconstrução.

A etapa da decomposição pode ser subdividida em incorporação e decomposição em valores singulares (SVD - Singular Value Decomposition).

Seja $Y_{T}=\left[y_{1}, \ldots, y_{T}\right] \in \mathbb{R}^{T}$ uma série temporal com cardinalidade igual a $T$ e $F: \mathbb{R}^{T} \rightarrow$ $\mathbb{R}^{L \times K}$ um mapa invertível. Por incorporação, entende-se como sendo um procedimento no qual uma série temporal $Y_{T} \in \mathbb{R}^{T}$ é transformada pelo mapa $F$ em uma matriz $X=\left[X_{1}, \ldots, X_{T}\right]_{L \times K} \in \mathbb{R}^{L \times K}$, onde $X_{k}=\left[y_{K}, \ldots, y_{K+L-1}\right]^{T} \in \mathbb{R}^{L}$, para todo $k$. Isto é, $Y_{T} \in \mathbb{R}^{T} \stackrel{F}{\rightarrow} X \in \mathbb{R}^{L \times K}$, onde $K=T-L+$ 1. A matriz $X$ é conhecida como matriz trajetória (HASSANI, 2007) e o parâmetro $L$, que assume algum valor inteiro no intervalo $2 \leq L \leq T$ é o tamanho da janela da matriz trajetória (GOLYANDINA et al., 2001). A matriz trajetória $X$ pode ser expandida via decomposição em valores singulares, em (1).

$$
X=\sum_{\lambda_{l} \in \sigma(S)}\left(\lambda_{l}\right)^{\frac{1}{2}} U_{l} V_{l}^{T}=\sum_{l=1}^{L} E_{l}
$$

Onde: $E_{l}:=\lambda_{l}{ }^{1 / 2} U_{l} V_{l}^{T}$ e os conjuntos $\left\{\lambda_{l}{ }^{1 / 2}\right\}_{l=1}^{L}$ e $\left\{U_{l}\right\}_{l=1}^{L}$ são, respectivamente, denominados por espectro singular e de vetores singulares da matriz trajetória $X$. A coleção $\left(\lambda_{l}, U_{l}, V_{l}\right)$ é conhecida como autotripla na $S V D$ da matriz trajetória $X$. A contribuição de cada componente em (1) pode ser mensurada pela razão de valores singulares, dada por $\left(\lambda_{1}\right)^{1 / 2} / \sum_{l=1}^{L}\left(\lambda_{1}\right)^{1 / 2}$. Considere que $d$ seja o posto (isto é, o número de autovalores não nulos) da matriz trajetória $X$. Segue que a identidade descrita em (1) pode ser reescrita tal como:

$$
X=\sum_{l=1}^{d} E_{l} \text {, onde } d \leq L
$$

A etapa de reconstrução pode ser subdividida em agrupamento e média diagonal. A etapa de agrupamento consiste no procedimento de agrupar algumas sequências de matrizes elementares resultantes da decomposição SVD em grupos disjuntos e, após isso, somá-las, gerando novas matrizes elementares.

Considerando a sequência $\left\{E_{l}\right\}_{l=1}^{d}$ de matrizes elementares na SVD, em (2). Agrupando-as em $m \leq d$ grupos disjuntos e assumindo que, após o agrupamento, o conjunto de índices gerado é dado por $\left\{I_{1}, \ldots, I_{m}\right\}$, onde, para todo $i, I_{i}=\left\{I_{i 1}, \ldots, I_{i p_{i}}\right\}$ e $p_{i}$ é a cardinalidade do grupo $I_{i}$, então a matriz elementar $X_{I_{i}}$ gerada a partir do grupo $\left\{X_{I_{i j}}\right\}_{j=1}^{p_{i}}$ é dada por $X_{I_{i}}=\sum_{j=1}^{p_{i}} X_{I_{i j}}$, de modo que a identidade em (2) pode ser reescrita como em (3).

$$
X=\sum_{i=1}^{m} X_{I_{i}}
$$

É importante salientar que o procedimento de agrupamento pode ser realizado também sob a sequência $\bigcup_{i=1}^{m}\left\{X_{I_{i j}}\right\}_{j=1}^{p_{i}}$ de igual forma ao realizado na sequência $\left\{E_{l}\right\}_{l=1}^{d} u$ tilizando, inclusive, 
um método ou critério de agrupamento diferente do aplicado sob a sequência $\left\{E_{l}\right\}_{l=1}^{d}$ em (2). Um dos objetivos da análise de agrupamentos é separar as componentes do sinal das componentes do ruído. A contribuição da componente $X_{I_{i}}$ pode ser mensurada pela razão de valores singulares dada $\operatorname{por} \sum_{j=1}^{p_{i}}\left(\lambda_{I_{i j}}\right)^{1 / 2} / \sum_{l=1}^{d}\left(\lambda_{1}\right)^{1 / 2}$.

Dada a matriz trajetória $X$ e assumindo que $L^{*}=\min (L, K)$ e que $K^{*}=\max (L, K)$. Considere que $x_{l, k}^{(i)}$ seja um elemento na linha $l$ e coluna $k$ na matriz $X_{I_{i}}$. O elemento $y_{t}^{(i)}$ da componente SSA $\left[y_{t}^{(i)}\right]_{1 \times T}$ é calculado por meio da média diagonal, que é definida em (4), a partir da matriz elementar $X_{I_{i}}$.

$$
y_{t}^{(i)}=\left\{\begin{array}{c}
\frac{\sum_{l=1}^{t} x_{l, t-l+1}^{(i)}}{t}, \text { se } 1 \leq t<L^{*} \\
\frac{\sum_{l=1}^{L^{*}} x_{l, t-l+1}^{(i)}}{L^{*}}, \text { se } L^{*} \leq t<K^{*} \\
\frac{\sum_{l=t-K^{*}+1}^{T-K^{*}+1} x_{l, t-l+1}^{(i)}}{T-K^{*}+1}, \text { se } K^{*} \leq t \leq T
\end{array}\right.
$$

Cada componente $\left[y_{t}^{(i)}\right]_{1 \times T}$ concentra parte da energia da série temporal original $\left[y_{t}\right]_{1 \times T}$ que pode ser mensurada pela razão de valores singulares $\sum_{j=1}^{p_{i}}\left(\lambda_{I_{i j}}\right)^{1 / 2} / \sum_{l=1}^{d}\left(\lambda_{1}\right)^{1 / 2}$

De acordo com GOLYANDINA et al. (2001), as componentes SSA $\left[y_{t}^{(i)}\right]_{1 \times T}$ podem ser classificadas em três categorias: tendência, componentes harmônicas e ruído.

Por correlação ponderada $(w)$, entende-se como sendo a função que quantifica a dependência linear entre duas componentes SSA $Y_{t}^{(i)}$ e $Y_{t}^{(j)}$, conforme definido por $\quad \rho_{i j}^{(w)}=$ $\frac{\left(Y_{t}^{(i)}, Y_{t}^{(j)}\right)_{w}}{\left\|Y_{t}^{(i)}\right\|_{w}\left\|Y_{t}^{(j)}\right\|_{w}}$, onde \|\| é a norma euclidiana, ()$_{w}$ é o produto interno tal que: $\left\|Y_{t}^{(i)}\right\|_{w}=$ $\sqrt{\left(Y_{t}^{(i)}, Y_{t}^{(i)}\right)_{w}}$ e $\left(Y_{t}^{(i)}, Y_{t}^{(j)}\right)_{w}=\sum_{k=1}^{T} w_{k} y_{k}^{(i)} y_{k}^{(j)} ; \mathrm{e} w_{k}=\min \{k, L, T-k\}$

Por meio da separabilidade, pode-se verificar estatisticamente se as duas componentes SSA estão bem separadas, em termos de dependência linear. Se o valor absoluto $\rho_{i j}^{(w)}$ é pequeno (HASSANI, 2007), então as componentes SSA correspondentes são classificadas como $w$ ortogonais (ou quase $w$-ortogonais); caso contrário, são ditas mal separadas.

A análise das coordenadas da série temporal na base definida pelos vetores singulares resultantes da SVD permite identificar as componentes de tendência e da sazonalidade da série. O problema geral aqui consiste em identificar e separar as componentes oscilatórias das componentes que fazem parte da tendência. De acordo com GOLYANDINA et al. (2001) a análise gráfica de tais coordenadas aos pares permite identificar por meio visual as componentes harmônicas da série.

As coordenadas da série temporal em duas componentes ortogonais podem ser dispostas em um diagrama de dispersão. Considere um harmônico puro com frequência igual a $\omega$, fase igual a $\delta$, amplitude igual a $\xi$ e período $\rho=\frac{1}{\omega}$ definido como um divisor do tamanho da janela $L$ e $K$. Se o parâmetro $\rho$ assume um valor inteiro, então $\rho$ é classificado como período do harmônico. Por exemplo, as funções seno e o cosseno com frequências, amplitudes e fases iguais resultam em um diagrama de dispersão que exibe um padrão circular. Por sua vez, se $\rho=\frac{1}{\omega}$ é um inteiro, então o 
diagrama de dispersão exibe um polígono regular $\operatorname{com} \rho$ vértices. Para uma frequência $\omega=m / n<$ 0.5 com $m$ e $n$ inteiros e primos, os pontos são vértices de um polígono regular de $n$ vértices (GOLYANDINA et al., 2001). Dessa forma, a identificação dos componentes que são gerados por um harmônico é reduzida à análise pictórica do padrão determinado nos diferentes pares de componentes.

\section{MULTI-CHANNEL SINGULAR SPECTRUM ANALYSIS}

MSSA é uma extensão do SSA para trabalhar com análise e previsão de séries temporais multidimensionais. O procedimento MSSA segue a mesma estrutura do procedimento SSA com a diferença de fazê-lo usando um conjunto de séries temporais em face de uma única série

Considere o sistema de $s$ séries temporais de tamanho $T$

$$
Y^{(k)}=\left(y_{t}^{(k)}\right)_{t=1}^{T}
$$

onde $k=1, \ldots, s$. O caso particular do procedimento MSSA para $s=1$, equivale ao procedimento usando SSA (GOLYANDINA et al., 2005).

Escolhendo um único tamanho de janela de defasagem $L$ para todas as $s$ séries, onde $1<L<T$, na fase de incorporação MSSA são obtidos $K=T-L+1$ vetores defasados $X_{j}^{(k)}=$ $\left(y_{j}^{(k)}, \ldots, y_{j+L-1}^{(k)}\right)^{T}, j=1, \ldots, K$ para cada série $Y^{(k)}, k=1, \ldots, s$. Assim, para cada série $Y^{(k)}$, é possível obter através de um mapa invertível $F^{(k)}$, uma matriz trajetória como em (6).

$$
X^{(k)}=\left[\begin{array}{cccc}
y_{1}^{(k)} & y_{2}^{(k)} & \cdots & y_{K}^{(k)} \\
y_{2}^{(k)} & y_{3}^{(k)} & \cdots & y_{K+1}^{(k)} \\
\vdots & \vdots & \ddots & \vdots \\
y_{L}^{(k)} & y_{L+1}^{(k)} & \cdots & y_{T}^{(k)}
\end{array}\right]
$$

A matriz trajetória da série multidimensional $\left(Y^{(1)}, Y^{(2)}, \ldots, Y^{(s)}\right)$ é, então, uma matriz de dimensão $L s \times K$ e tem a seguinte forma:

$$
X=\left[X_{1}^{(1)}: \ldots: X_{K}^{(1)}: \ldots: X_{1}^{(s)}: \ldots: X_{K}^{(s)}\right]^{T}=\left[X^{(1)}: \ldots: X^{(s)}\right]^{T}
$$

O espaço trajetória (GOLYANDINA et al. (2010)) é definido por um espaço linear spanado pelos vetores defasados (colunas da matriz trajetória $X$ ).

A partir de $S=X X^{T}$, onde os autovalores de $S$ em ordem de magnitude $\lambda_{1} \geq \cdots \geq \lambda_{L s} \geq 0$, $U_{1}, \ldots, U_{L S}$ os respectivos autovalores associados, $d=\max \left\{j: \lambda_{j}>0\right\}$ o posto da matriz $S$ e $V_{J}=$ $\frac{X^{T} U_{j}}{\sqrt{\lambda_{j}}}, j=1, \ldots, d$. Denotando $E_{j}=\sqrt{\lambda_{j}} U_{j} V_{j}^{T}$, então o SVD da matriz trajetória $X$ pode ser escrito por: $X=E_{1}+\cdots+E_{d}$.

De forma análoga, a fase de agrupamento particiona o conjunto de índices $\{1, \ldots, d\}$ em $m$ subconjuntos disjuntos $I_{1}, \ldots, I_{m}$ de modo que a matriz trajetória seja reescrita na forma conhecida como decomposição agrupada:

$$
X=X_{I_{1}}+\cdots+X_{I_{m}}
$$


Por fim a média diagonal é aplicada a cada uma das séries decomposta em (8) e então o grupo de sinais reconstruídos dado por $\tilde{Y}^{(k)}=\left(\tilde{Y}_{t}^{(k)}\right)_{t=1}^{T}, k=1, \ldots, s$ é obtido.

\section{MODELOS AUTORREGRESSIVOS PERIÓDICOS}

Segundo HIPEL \& McLEOD (1994), algumas séries temporais têm uma estrutura de autocorrelação que depende não somente do intervalo de tempo entre as observações, mas também do período observado. Estas séries podem ser analisadas por formulações autoregresivas cujos parâmetros têm comportamento periódico. Estes modelos são chamados de auto-regressivos periódicos $\operatorname{PAR}(p)$ onde $p$ é um vetor apresentado por $p=\left(p_{1}, p_{2}, \ldots, p_{s}\right), s$ é o período considerado: se for mensal, $s=12$, se for trimestral, $s=4$. Para os estudos desta proposta serão considerados períodos mensais. O modelo $\operatorname{PAR}(p)$ pode ser representado através da padronização das observações no modelo $\operatorname{AR}(p)$ como em (9).

$$
\begin{aligned}
\left(\frac{Y_{t}-\mu_{m}}{\sigma_{m}}\right)= & \varphi_{1}^{m}\left(\frac{Y_{t-1}-\mu_{m-1}}{\sigma_{m-1}}\right)+\varphi_{2}^{m}\left(\frac{Y_{t-2}-\mu_{m-2}}{\sigma_{m-2}}\right)+\cdots+\varphi_{p_{m}}^{m}\left(\frac{Y_{t-p_{m}}-\mu_{m-p_{m}}}{\sigma_{m-p_{m}}}\right) \\
& +a_{t}^{m}
\end{aligned}
$$

onde $Y_{t}$ é a série sazonal de período $s=12, t=1, \ldots, T, m=1, \ldots, s, \mu_{m}$ é a média sazonal de período $s, p_{m}$ é a ordem do operador autorregressivo de período $m$ - neste caso, a ordem muda de acordo com o período, e $a_{t}^{m}$ é a série de resíduos independentes e identicamente distribuídos com média zero e variância $\sigma_{a}^{2(m)}$.

A ideia consiste no ajuste de um modelo autorregressivo de ordem $p_{m}$ para cada um dos meses da série original. A primeira etapa consiste na identificação das ordens $p_{m}$ mais apropriadas aos operadores autorregressivos de cada período. Esta etapa pode ser realizada a partir das funções de autocorrelação e de autocorrelação parcial.

A estrutura de dependência temporal da série pode ser descrita pelo conjunto de funções de autocorrelação $\rho_{k}^{m}$ dos períodos $m=1,2 \ldots, s$ :

$$
\begin{aligned}
\rho_{k}^{m}=E\left[\left(\frac{Y_{t}-\mu_{m}}{\sigma_{m}}\right)\left(\frac{Y_{t-k}-\mu_{m-k}}{\sigma_{m-k}}\right)\right] \\
=\varphi_{1}^{m} E\left[\left(\frac{Y_{t}-\mu_{m-1}}{\sigma_{m-1}}\right)\left(\frac{Y_{t-k}-\mu_{m-k}}{\sigma_{m-k}}\right)\right]+\cdots \\
+\varphi_{p_{m}}^{m} E\left[\left(\frac{Y_{t}-\mu_{m-p_{m}}}{\sigma_{m-p_{m}}}\right)\left(\frac{Y_{t-k}-\mu_{m-k}}{\sigma_{m-k}}\right)\right] \\
+E\left[a_{t}^{m}\left(\frac{Y_{t-k}-\mu_{m-k}}{\sigma_{m-k}}\right)\right]
\end{aligned}
$$

Fixando $m$ e variando $k$ de 1 a $p_{m}$ em (10) obtém-se para cada período um conjunto de equações periódicas de Yule-Waker.

$$
\left[\begin{array}{cccc}
1 & \rho_{1}^{m-1} & \cdots & \rho_{k-1}^{m-1} \\
\rho_{1}^{m-1} & 1 & \cdots & \rho_{k-2}^{m-2} \\
\vdots & \vdots & \ddots & \vdots \\
\rho_{k-1}^{m-1} & \rho_{k-2}^{m-2} & \cdots & 1
\end{array}\right]\left[\begin{array}{c}
\varphi_{k 1}^{m} \\
\varphi_{k 2}^{m} \\
\vdots \\
\varphi_{k k}^{m}
\end{array}\right]=\left[\begin{array}{c}
\rho_{k 1}^{m} \\
\rho_{k 2}^{m} \\
\vdots \\
\rho_{k k}^{m}
\end{array}\right]
$$


O conjunto de valores $\varphi_{k k}^{m}, m=1, \ldots, s$ é denominado autocorrelação parcial do período $m$. Cada coeficiente de autocorrelação parcial de ordem $k$ coincide com o último parâmetro de um modelo autorregressivo da mesma ordem. Assim, em um processo autorregressivo de ordem $p_{m}$, a função de autocorrelação parcial $\varphi_{k k}^{m}$ é diferente de zero para $k$ menor ou igual a $p_{m}$ e zero para $k$ maior que $p_{m}$. Portanto, a identificação clássica do modelo $\operatorname{PAR}(p)$ fundamenta-se em determinar as ordens apropriadas aos operadores autorregressivos de cada período $p_{m}, m=1, \ldots, s$. Estas ordens são determinadas de acordo com as estimativas $\hat{\varphi}_{k k}^{m}, k=1, \ldots, T / 4 \mathrm{e}$ substituindo as autocorrelações pelos respectivos valores amostrais em (11). Se a ordem do operador autorregressivo em um determinado período $m$ for igual a $p_{m}$, então $\hat{\varphi}_{k k}^{m}$ terá distribuição aproximadamente normal com média zero e variância $1 / T$ quando $k>p_{m}$. Na ocasião, procura-se a maior ordem $i$ para cada período $m$ de modo que todas as estimativas $\hat{\varphi}_{k k}^{m}$ não sejam mais significativas para $k>i$.

Os parâmetros $\varphi_{i}^{m}, \quad i=1, \ldots, p_{m}$ são estimados substituindo os parâmetros $\rho_{j}^{m-k}, j=$ $0, \ldots,\left(p_{m}-1\right), k=1, \ldots, p_{m}$ por suas medidas amostrais em (11).

\section{GERAÇÃO DE CENÁRIOS}

Nesta seção são apresentados conceitos, formulação matemática e estatísticas utilizadas na geração de cenários na forma como são implementadas no modelo Newave (CEPEL, 2000). O valor observado da série histórica no instante $t$ pode ser interpretado como um valor amostrado da distribuição de probabilidade associada a variável aleatória do processo estocástico em $t$. Como não há disponível todas as ocorrências do processo estocástico, o objetivo de ajustar o modelo $\operatorname{PAR}(p)$ para que se tenha o gerador da série histórica e, a partir dele, gerar séries sintéticas que representem as séries temporais possíveis de serem amostradas pelo processo. O modelo PAR ajustado deve permitir então que se façam tantos sorteios quanto forem necessários para o problema em questão. Assim, cada sorteio estará associado a uma série sintética.

À partir da equação (10) pode-se chegar à expressão:

$$
\begin{aligned}
& a_{t}^{m}>-\frac{\mu_{m}}{\sigma_{m}}-\varphi_{1}^{m}\left(\frac{Y_{t-1}-\mu_{m-1}}{\sigma_{m-1}}\right)-\varphi_{2}^{m}\left(\frac{Y_{t-2}-\mu_{m-2}}{\sigma_{m-2}}\right)-\cdots \\
- & \varphi_{p_{m}}^{m}\left(\frac{Y_{t-p_{m}}-\mu_{m-p_{m}}}{\sigma_{m-p_{m}}}\right)
\end{aligned}
$$

Nomeando o lado direito de (12) por $\Delta$, temos:

$$
a_{t}^{m}>\Delta
$$

Com isso, vemos que $\Delta$ é função apenas dos dois primeiros momentos do período $m$ e dos coeficientes autorregressivos e é dada por:

$$
\begin{aligned}
\Delta=-\frac{\mu_{m}}{\sigma_{m}}- & \varphi_{1}^{m}\left(\frac{Y_{t-1}-\mu_{m-1}}{\sigma_{m-1}}\right)-\varphi_{2}^{m}\left(\frac{Y_{t-2}-\mu_{m-2}}{\sigma_{m-2}}\right)-\cdots \\
- & \varphi_{p_{m}}^{m}\left(\frac{Y_{t-p_{m}}-\mu_{m-p_{m}}}{\sigma_{m-p_{m}}}\right)
\end{aligned}
$$

Muitos pesquisadores assumem que os resíduos $a_{t}^{m}$ apresentam distribuição normal e uma possível não normalidade pode ser corrigida pela transformação Box-Cox (Box E Cox, 1964). O modelo de geração de séries sintéticas deve ser aplicado diretamente a série temporal original sem quaisquer transformação para torná-la estacionária e deve ser capaz de lidar com resíduos que apresentam um forte coeficiente de assimetria. Para isso foi adotado o ajuste de uma distribuição log-normal com três parâmetros aos resíduos mensais $a_{t}^{m}$ (MACEIRA, 1989). Assim a variável $\xi_{t}$ segue uma distribuição normal com média $\mu_{\xi_{t}}$ e variância $\sigma_{\xi_{t}}^{2(m)}, a_{t}=e^{\xi_{t}}+\Delta$ e $a_{t} \sim \operatorname{LNormal}\left(\mu_{\xi_{t}}, \sigma_{\xi_{t}}^{2(m)}, \Delta\right)$. Assim: $\xi_{t}=\ln \left(a_{t}-\Delta\right)$. 
Para avaliar o desempenho do modelo em relação à geração de cenários, alguns testes são realizados com vistas a avaliar a capacidade de reproduzir as propriedades estatísticas da série original. Dentre eles se destacam os testes da Média (CASELA \& BERGER, 2010), o Teste de Levene para variâncias (ALMEIDA \& ELLIAN, 2008), o Teste de aderência de KolmogorovSmirnov (ARANGO, 2005) e a análise de sequências que avalia o comprimento, a soma e a intensidade da sequências de períodos críticos de baixa velocidade do vento que os cenários são capazes de detectar.

\section{METODOLOGIA PROPOSTA}

A metodologia a ser utilizada consiste basicamente em modelar via $\operatorname{PAR}(p)$ duas séries de médias mensais de velocidade do vento após uma filtragem MSSA para em seguida gerar cenários conforme indicado na seção 5. Para a escolha do parâmetro $L$ em MSSA, é usado o teste BDS (BROCK et. al, 1987) de modo que à partir de $L=T / 2$, procede-se com a filtragem SSA/MSSA, decompondo as séries em sinal e ruído. Aplica-se o teste BDS à série de ruídos obtida e, caso a hipótese nula de dados não correlacionados não for rejeitada a um nível de 5\% de significância nas 6 dimensões medidas pelo teste, procede-se com a modelagem $\operatorname{PAR}(p)$ da série de sinal obtida na decomposição em SSA/MSSA. Caso o teste rejeite a hipótese nula, procede-se com a subtração de uma unidade do comprimento de janela $L=T / 2-1$ e novamente a filtragem SSA/MSSA é feita até que a hipótese nula não seja rejeitada.

Após a filtragem, a modelagem é feita através da equação (15).

$$
\left(\frac{\hat{Y}_{t}-\hat{\mu}_{m}}{\hat{\sigma}_{m}}\right)=\varphi_{1}^{m}\left(\frac{\hat{Y}_{t-1}-\hat{\mu}_{m-1}}{\hat{\sigma}_{m-1}}\right)+\cdots+\varphi_{p_{m}}^{m}\left(\frac{\hat{Y}_{t-p_{m}}-\hat{\mu}_{m-p_{m}}}{\hat{\sigma}_{m-p_{m}}}\right)+a_{t}^{m},
$$

onde $\hat{Y}_{t}$ é a série sazonal filtrada MSSA de período $s=12, t=1, \ldots, T, m=1, \ldots, s, \hat{\mu}_{m}$ é a média sazonal filtrada SSA/MSSA de período $s, p_{m}$ é a ordem do operador autorregressivo de período $m$ - neste caso, a ordem muda de acordo com o período -, e $a_{t}^{m}$ é a série de resíduos independentes e identicamente distribuídos com média zero e variância $\sigma_{a}^{2(m)}$.

Para a geração de cenários, a série de ruídos excluída na modelagem é realocada através do processo bootstrap para que os desvios padrão dos cenários fiquem bem mais próximos do desvio histórico. Neste caso as séries de ruídos de comprimento 192 são distribuídas em séries de comprimento 16 para cada mês.

Os cenários são concatenados mês a mês gerando 60 meses de horizonte. Para ambas as séries são gerados 200 cenários e o desempenho dos mesmos são testados via Teste de Levene para a variância, teste $t$ Student para a média e teste de sequências negativas para avaliar a capacidade de reprodução de períodos críticos destes modelos.

\section{ESTUDO DE CASO}

Nos experimentos foram considerados os registros de velocidade do vento provenientes das estações anemométricas do Instituto Nacional de Meteorologia - INMET (http://www.inmet.gov.br/portal/index.php?r=bdmep/bdmep) localizadas nas cidades pernambucanas de Petrolina e Pesqueira. Os registros analisados abrangem o período de janeiro de 1996 até dezembro de 2012. A figura 1 apresenta o comportamento destas duas séries. 


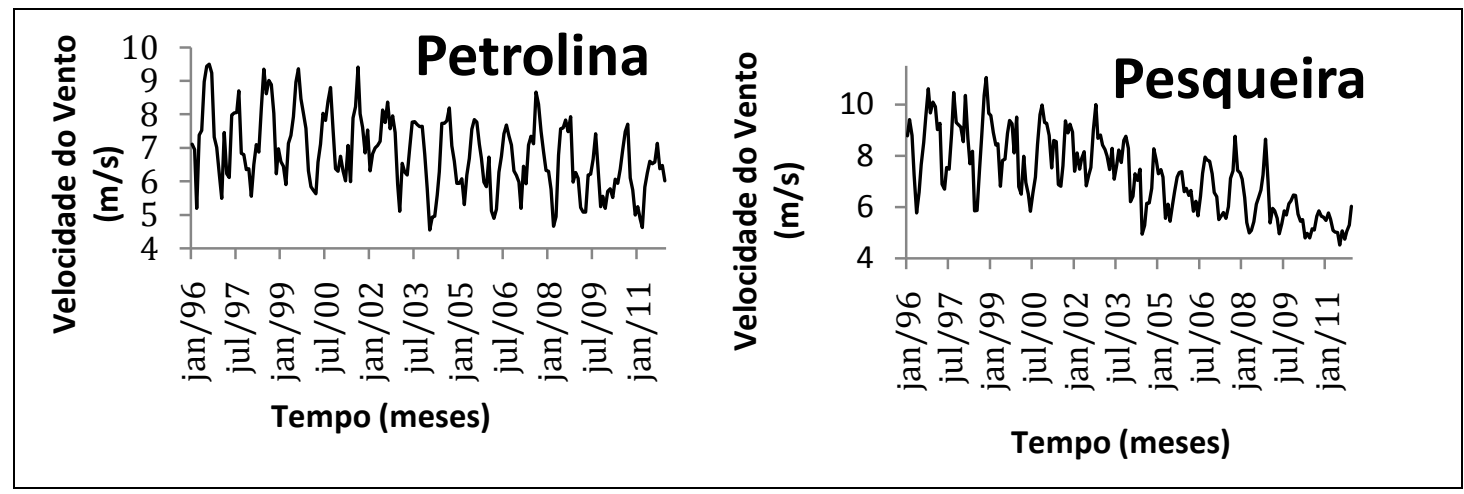

Figura 1. Séries originais de médias mensais de velocidade do vento nas Estações Anemométricas de Petrolina e Pesqueira (PE).

Inicialmente, procede-se com a filtragem simultânea das séries para em seguida modelar via PAR(p). A matriz trajetória multidimensional da fase de incorporação MSSA tem dimensão $(2 L \times K)$. Neste caso, cada série tem dimensão $T=192$ e o tamanho de janela utilizado foi $L=96$, logo a matriz trajetória multidimensional tem dimensão $192 \times 97$. Após o processo MSSA, as duas séries são decompostas em tendência, componente harmônica e ruído. A matriz de correlação ponderada apresentada na tabela 1 confirma a separabilidade entre estas componentes.

Tabela 1. Correlação Ponderada entre as componentes MSSA.

\begin{tabular}{c|c|c|c}
\hline Componentes & Tendência & Harmônica & Ruído \\
\hline Tendência & 1 & 0,001 & 0,003 \\
Harmônica & 0,001 & 1 & 0,029 \\
Ruído & 0,003 & 0,029 & 1 \\
\hline
\end{tabular}

O teste BDS, aplicado as componentes ruidosas de cada série confirma a hipótese de séries de ruídos e as mesas são excluídas para que as séries reconstruídas com as componentes de tendência e harmônica sejam modeladas para geração de cenários. A tabela 2 mostra os resultados do teste BDS aplicados a estas séries.

Tabela 2. Testes BDS aplicados às séries de ruídos.

\begin{tabular}{c|c|c|c|c|c}
\hline & Dim & Estatística BDS & Estatística $Z$ & Erro padrão & $p$-valor \\
\cline { 2 - 6 } Petrolina & 2 & 0,006156 & 1,379423 & 0,004463 & 0,1678 \\
& 3 & 0,005718 & 0,805545 & 0,007098 & 0,4205 \\
& 4 & 0,004280 & 0,506136 & 0,008457 & 0,6128 \\
& 5 & 0,004037 & 0,457835 & 0,008817 & 0,6471 \\
& 6 & 0,000435 & 0,051183 & 0,008504 & 0,9592 \\
\hline \multirow{5}{*}{ Pesqueira } & 3 & 0,001376 & 1,114620 & 0,001234 & 0,2650 \\
& 4 & 0,001597 & 0,795097 & 0,002008 & 0,4266 \\
& 5 & 0,001581 & 0,553033 & 0,002859 & 0,5802 \\
& 6 & 0,005030 & 1,336379 & 0,003764 & 0,1814 \\
\hline
\end{tabular}

A mesma análise feita com a filtragem MSSA foi feita com as séries separadamente via SSA e a modelagem PAR(p) foi feita para os três casos: as séries originais sem a filtragem; as séries com as filtragens SSA individuais e as séries filtradas via MSSA. Para avaliar o poder preditivo dos modelos, a medida estatística de erro MAPE (Mean Absolute Pencentage Error) dada por: 


$$
M A P E=\frac{100}{T_{h}} \sum_{t=1}^{T_{h}} \frac{\left|y_{t}-\hat{y}_{t}\right|}{y_{t}}
$$

onde $y_{t}$ and $\hat{y}_{t}$ são respectivamente os valores originais e previstos de velocidade do vento no período $t$ e $T_{h}$ é o horizonte de previsão, foi calculada em cada um dos três casos. Os resultados estão apresentados na tabelas 3 .

Tabela 3. MAPE $(\%)$ in sample dos meses para os modelos $\operatorname{PAR}(p)$,

$\operatorname{PAR}(p)$ - SSA e PAR $(p)$ - MSSA nas Estações Petrolina e Pesqueira.

\begin{tabular}{l|c|c|c|c|c|c}
\hline \multicolumn{1}{c|}{ MAPE } & \multicolumn{3}{|c|}{ PETROLINA } & \multicolumn{3}{c}{ PESQUEIRA } \\
\hline Mês & PAR $(p)$ & $\begin{array}{l}\text { PAR }(p) \\
- \text { SSA }\end{array}$ & $\begin{array}{l}\text { PAR }(p)- \\
\text { MSSA }\end{array}$ & PAR $(p)$ & $\begin{array}{l}\text { PAR }(p)- \\
\text { SSA }\end{array}$ & $\begin{array}{l}\text { PAR }(p)- \\
\text { MSSA }\end{array}$ \\
\hline Janeiro & 6,1200 & 3,3072 & $\mathbf{1 , 4 8 2 7 0}$ & 6,1182 & 4,7278 & $\mathbf{3 , 5 4 1 3}$ \\
Fevereiro & 8,7405 & 3,1330 & $\mathbf{0 , 8 7 9 0 4}$ & 5,1540 & 2,6814 & $\mathbf{1 , 1 2 1 1}$ \\
Março & 6,1584 & 4,1720 & $\mathbf{0 , 8 1 2 4 7}$ & 7,1102 & 1,9755 & $\mathbf{1 , 4 7 8 1}$ \\
Abril & 5,9539 & 2,4138 & $\mathbf{0 , 6 3 0 7 1}$ & 7,4127 & 3,1508 & $\mathbf{1 , 8 2 1 6}$ \\
Maio & 4,4594 & 3,3287 & $\mathbf{0 , 5 1 4 7 8}$ & 5,7543 & 2,7266 & $\mathbf{1 , 7 5 1 2}$ \\
Junho & 4,5123 & 2,1882 & $\mathbf{0 , 5 1 5 9 2}$ & 4,3649 & 2,4026 & $\mathbf{1 , 3 5 9 0}$ \\
Julho & 2,9755 & 1,9037 & $\mathbf{0 , 5 4 7 3 9}$ & 3,8340 & 1,9483 & $\mathbf{1 , 4 1 6 5}$ \\
Agosto & 3,5905 & 2,3111 & $\mathbf{0 , 6 1 3 1 6}$ & 4,5714 & 2,2650 & $\mathbf{1 , 7 5 6 7}$ \\
Setembro & 5,0779 & 2,5236 & $\mathbf{0 , 7 0 4 1 8}$ & 5,3439 & 1,7724 & $\mathbf{1 , 4 1 9 3}$ \\
Outubro & 5,6350 & 2,4985 & $\mathbf{0 , 7 3 2 3 7}$ & 5,9651 & 2,1032 & $\mathbf{1 , 4 0 8 6}$ \\
Novembro & 4,1773 & 1,9260 & $\mathbf{0 , 6 2 4 0 8}$ & 7,0626 & 2,3336 & $\mathbf{1 , 2 8 6 9}$ \\
Dezembro & 4,3738 & 2,5884 & $\mathbf{0 , 6 8 4 3 7}$ & 4,4704 & 0,8727 & 1,0379 \\
\hline
\end{tabular}

Na geração de cenários espera-se que o comportamento dos cenários utilizando os modelos $\operatorname{PAR}(p)$ obtidos sem e com as filtragens SSA/MSSA seja idêntico ao comportamento das séries históricas. Esta verificação é feita testando a igualdade entre as médias e entre as variâncias em cada um dos 60 meses de cenários gerados em comparação com a série histórica. Os resultados destes testes estão apresentados nas tabelas 4,5 e 6 . Sendo as tabelas 4 e 5 os percentuais de não rejeição da hipótese nula de igualdade entre as médias e as variâncias, respectivamente, e a tabela 6 , os resultados do teste de sequências negativas.

Tabela 4. teste $t$-Student em comparação com a série histórica.

\begin{tabular}{l|c|c|c}
\hline & \multicolumn{3}{|c}{ Não Rejeita $\mathrm{H}_{0}(\%)$} \\
\hline Estação Anemométrica & PAR(p) & PAR(p) - SSA & PAR(p) - MSSA \\
\hline Petrolina & 100 & 95 & 90 \\
Pesqueira & 90 & 80 & 85 \\
\hline
\end{tabular}

Tabela 5. Teste de Levene em comparação com a série histórica.

\begin{tabular}{l|c|c|c}
\hline & \multicolumn{3}{|c}{ Não Rejeita $\mathrm{H}_{0}(\%)$} \\
\hline Estação Anemométrica & PAR(p) & PAR(p) - SSA & PAR(p) - MSSA \\
\hline Petrolina & 100 & 99 & 94 \\
Pesqueira & 99 & 99 & 95 \\
\hline
\end{tabular}


Tabela 6. Teste de sequências negativas.

\begin{tabular}{ccccc}
\hline & Estação & $\begin{array}{c}\text { Comprimento } \\
\text { Anemométrica }\end{array}$ & $\begin{array}{c}\text { Soma } \\
\text { Valor Crítico: } 3,84\end{array}$ & $\begin{array}{c}\text { Intensidade } \\
\text {-valor min: } 0,05\end{array}$ \\
$p$ - valor min: 0,05 \\
\hline \multirow{2}{*}{ PAR(p) } & Petrolina & 1,04 & 0,1206 & 0,2906 \\
& Pesqueira & 0,17 & 0,9355 & 0,8842 \\
\hline PAR(p) & Petrolina & 2,16 & 0,3840 & 0,8376 \\
-SSA & Pesqueira & 0,36 & 0,6170 & 0,3226 \\
\hline PAR(p) & Petrolina & 5,78 & 0,1210 & 0,5450 \\
-MSSA & Pesqueira & 0,27 & 0,2046 & 0,1820 \\
\hline
\end{tabular}

\section{CONCLUSÕES}

Este artigo apresentou a proposta de geração de cenários de velocidade do vento a partir da modelagem PAR(p) com filtragem SSA e MSSA em duas estações anemométricas localizadas no Nordeste do Brasil. Para as filtragens SSA e MSSA, o teste BDS aplicado às séries de ruídos mostra que elas são independentes até a sexta dimensão. Isso garante a princípio que as séries que estão sendo removidas nas filtragens são ruídos e não uma parte do sinal. Outra medida utilizada foi a correlação ponderada entre as componentes de tendência, harmônica e ruído extraídas das séries. As medidas obtidas da correlação ponderada mostram que estas componentes estão bem separadas. Isto faz com que parte do sinal não seja removido como ruído ou parte de ruído não seja utilizado como sinal.

Após as filtragens SSA e MSSA, as séries foram modeladas via PAR(p), tanto as séries originais quando as séries filtradas. Com isso, foram ajustados modelos PAR(p) às séries de velocidade do vento antes e após a filtragem via SSA e MSSA. As medidas MAPE e RMSE mostram que a filtragem SSA antes da modelagem $\operatorname{PAR}(p)$ aumenta a acurácia das previsões e que a filtragem MSSA antes desta modelagem supera os resultados verificados anteriormente com a filtragem SSA. Por fim, os resultados apresentados nas tabelas 4, 5 e 6 mostram que os cenários gerados a partir do modelo PAR(p) com a abordagem SSA/MSSA são capazes de reproduzir as séries históricas. $\mathrm{O}$ estudo deste artigo também direciona na possibilidade de usar a modelagem PAR(p) na previsão de velocidade do vento e geração de cenários eólicos objetivando a inclusão deste tipo de geração de energia no Sistema Energético Brasileiro.

\section{REFERÊNCIAS BIBLIOGRÁFICAS}

[1] Almeida, A. \& Ellian, S. N. J. (2008) Modificações e Alternativas aos testes de Levene e de Brown e Forsythe para Igualdade de Variâncias e Médias. Revista Colombiana de Estadística, v 13, 2, pp. 241-260.

[2] Arango, H. G. (2005) Bioestatística Teórica e Computacional. 2a. Edição. Guanabara Koogan Gen editoras. Rio de Janeiro.

[3] BROCK, W. A., DECHERT, W., \& SCHEINKMAN, J. (1987). A test for independence based on the correlation dimension. Working paper, University of Winconsin at Madison, University of Houston, and University of Chicago.

[4] Casela, G. \& Berger, R. L. (2010) Inferência Estatística. Cengage Learning, São Paulo.

[5] CEPEL - Centro de Pesquisa de Energia Elétrica (2000) Manual de Referencia do Modelo Newave. RelatórioTécnico, Rio de Janeiro. 
[6] Elshorbagy, A.; Simonovic, S.P.; Panu, U.S. (2002) Noise reduction in chaotic hydrologic time series: facts and doubts, Journal of Hydrology, 256, 147-265.

[7] Golyandina, N., Nekrutkin, V., Zhihgljavsky, A. (2001). Analysis of time series structure: SSA and reletade techniques. Chapman\&Hall/CRC. New York, USA.

[8] Golyandina, N., Stepanov. D. (2005). SSA-based approaches to analysis and forecast of multidimensional time series.Proceedings of the Fifth Workshop on Simulation.Department of Mathematics, St. Petersburg State University, Russia.P. 293-298.

[9] Golyandina, N. (2010). On the choice of parameters in Singular Spectrum Analysis and related subspacebased methods. Statistics and Its Interface, 2010, 3, 259-279.

[10] Hassani, H. (2007) Golyandina, N. (2010). Singular Spectrum Analysis: Methodology and Comparison. Journal of Data Science. 5, 239-257.

[11] Hassani, H. .; Zokaei, M.; Von Rosen, D.; Amiri, S.; Ghodsi, M. (2009). Does noise reduction matter for curve fitting in growth curve models? Computer Methods and Programs in Biomedicine, 96, pp. 173-181.

[12] Hanson, R.T.; Dettinger, M.D.; Newhouse, M.W. (2006). Relations between climatic variability and hydrologic time series from four alluvial basins across the southwestern United States, Hydrogeology Journal.

[13] Hipel, K. W. e McLeod, A. I. (1994) Time Series Modelling of Water Resources and Environmental Systems.Amsterdam, The Netherlands: Elsevier.

[14] Jayawardena, A.W.; Gurung, A.B. (2000) Noise reduction and prediction of hydrometeorological time series: dynamical systemas approach vs. stochastic approach, Journal of Hydrology, 228, 242-264.

[15] Maceira, M. E. P. (1989) Operação Ótima de Reservatórios com Previsão de Afluências. Dissertação de Mestrado. COOPE/UFRJ, Rio de Janeiro.

[16] Maceira, M. E. P.; Penna, D. D. J..; Damázio, J. M. (2006). Geração de cenários sintéticos de energia e Vazão para o planejamento da operação energética, Cadernos do IME - Série Estatística, UERJ, Rio de Janeiro, pp. 11-35.

[17] Schreiber, T.; Grassberger, P. (1991) A simple noise-reduction method for real data, Phys Letter A, 160 (5), 411-418.

[18] Sivakumar, B.; Phoon, K.; Liong, S.; Liaw, C. (1999) A systematic approach to noise reduction in chaotic hydrological time series, Journal of Hydrology, 219, pp. 103-135. 